\title{
Quantitative comparison of cleavage and quasi-cleavage fracture surfaces in hydrogen embrittled low-carbon steel
}

\author{
E. D. Merson ${ }^{\dagger, 1}$, V. A. Poluyanov ${ }^{1}$, P. N. Myagkikh ${ }^{1}$, D. L. Merson ${ }^{1}$, A. Yu. Vinogradov ${ }^{2}$ \\ †Mersoned@gmail.com \\ ${ }^{1}$ Institute of Advanced Technologies, Togliatti State University, 14 Belorusskaya St., Togliatti, 445667, Russia \\ ${ }^{2}$ Department of Mechanical and Industrial Engineering, Norwegian University of Science and Technology, NTNU, \\ Trondheim, N-7491, Norway
}

\begin{abstract}
The formation mechanism of the quasi-cleavage (QC) fracture surface in hydrogen embrittled low-carbon steels is not fully understood and is, therefore, intensively debated in the literature. At present, there are two conflicting points of view regarding the origin of the fracture surfaces of this kind. The followers of the first concept argue that QC results from the true cleavage (TC) fracture facilitated by hydrogen. Their opponents believe that the QC surface forms through the modified ductile crack growth mechanism. To clarify the nature of the fracture mechanism of the hydrogen embrittled low-carbon steels, we compare the quantitative characteristics of the TC and QC facets belonging to the same fracture surface. To this end, the through-notched specimen of the annealed low-carbon steel grade S235JR was tensile tested under electrolytic in-situ hydrogen charging to the certain strain, at which the sizable length of the QC crack has been reached. After that, the specimen was broken up in liquid nitrogen. As a result, the fracture surface containing both QC and TC facets with a clear boundary between the two fracture modes with distinct morphologies was obtained. Using the confocal laser scanning microscopy (CLSM), it was shown that the relief of the QC fracture surface part is much more flat than the TC one. Using the CLSM topographic maps obtained from the fracture surface, as well as the original computerized method of quantitative facets analysis, it was found that the average misorientation angle of QC facets is 1.5 times lower, while curviness of these facets is 3 times higher in comparison to those characteristics of the TC facets. On the basis of the obtained results, it was concluded that the QC crack path and, thus, its propagation mechanism, should be significantly different from the path and mechanism of the TC crack propagation.
\end{abstract}

Keywords: hydrogen embrittlement, quantitative fractography, quasi-cleavage, confocal laser scanning microscopy, steel.

УДК: 538.911

\section{Количественное сравнение поверхностей разрушения}

\section{с морфологией скола и квазискола в охрупченной водородом низкоуглеродистой стали}

\author{
Мерсон Е. Д. ${ }^{\dagger, 1}$, Полуянов В. А. ${ }^{1}$, Мягких П. Н. ${ }^{1}$, Мерсон Д. Л. ${ }^{1}$, Виноградов А. Ю. ${ }^{2}$ \\ ${ }^{1}$ Научно-исследовательский институт прогрессивных технологий, Тольяттинский государственный университет, \\ ул. Белорусская, 14, Тольятти, 445667, Россия \\ ${ }^{2}$ Кафедра механики и промышленности, Норвежский технологический университет, НТУ, \\ Тронхейм, N-7491, Норвегия
}

\begin{abstract}
Механизм образования поверхности разрушения с морфологией квазискола (КС) в охрупченных водородом низкоуглеродистых сталях до конца не изучен и активно обсуждается в литературе. В настоящий момент существует две прямо противоположных точки зрения относительно происхождения изломов такого вида. Согласно первой, КС является результатом истинного скола (ИС), облегченного под действием водорода. По второй версии КС образуется в результате роста трещины по видоизмененному механизму вязкого разрушения. Для прояснения природы механизма разрушения низкоуглеродистых сталей охрупченных водородом в настоящей работе проведено сравнение количественных характеристик фасеток скола и квазискола в одном и том же изломе. Для этого надрезанный образец отожженной низкоуглеродистой стали марки S235JR растягивали при электролитическом in-situ наводороживании до заданной деформации, при которой трещина КС достигала необходимой длины. После чего образец доламывали в жидком азоте. В результате был получен излом, содержащий как фасетки КС, так и фасетки ИС с четкой границей между двумя частями излома, представленными данными видами морфологий. При помощи
\end{abstract}


конфокальной лазерной сканирующей микроскопии (КЛСМ) показано, что в масштабе нескольких фасеток рельеф участка излома с морфологией КС существенно более плоский по сравнению с участком ИС. С использованием топографических карт изломов, полученных при помощи КЛСМ, а также оригинальной компьютеризированной методики количественного анализа фасеток было установлено, что средний угол разориентировки фасеток КС в 1.5 раза ниже, а их кривизна (отклонение от плоскости) в 3 раза выше, чем у фасеток ИС. На основе полученных результатов сделан вывод о том, что путь трещин КС, а значит и механизм их роста должен существенно отличаться от пути и механизма распространения трещин ИС.

Ключевые слова: водородная хрупкость, количественная фрактография, квазискол, конфокальная лазерная сканирующая микроскопия, сталь.

\section{1. Введение}

Водородная хрупкость (BХ) ежегодно приводит к большому количеству отказов оборудования и аварий техногенного характера во многих отраслях промышленности, включая нефтегазовую, химическую, автомобильную, авиакосмическую и другие. Основной причиной ВХ считается атомарный водород, который, растворяясь в металлах и сплавах, вызывает ухудшение их механических свойств. Происходит это из-за того, что атомарный водород некоторым образом снижает напряжения необходимые для зарождения и роста трещин и, таким образом, вызывает водородо-ассистированное растрескивание (ВАР, hydrogen-assisted cracking) [1]. В низкоуглеродистых сталях и чистом железе с ОЦК решеткой ВАР приводит к образованию изломов специфического вида, называемых «квазисколом» (КС) [2 -9]. Для данных материалов, имеющих относительно простую ферритную микроструктуру, такой тип излома нехарактерен и наблюдается только в условиях BX и, по всей видимости, при жидкометаллическом охрупчивании [1]. В то время как нормальным механизмом хрупкого разрушения для них является истинный скол (ИС), который активируется, например, при хладноломкости $[3,7,10]$. Несмотря на большой накопленный объем экспериментальных данных и множество предложенных моделей и теорий, до сих пор существуют прямо противоположные точки зрения относительно механизма ВАР, ответственного за формирование поверхности КС.

Отличительной особенностью фасеток КС является наличие на их поверхности большого количества различных элементов вязкого разрушения, таких как гребни отрыва, наноразмерные ямки, следы скольжения [1]. Данная особенность, а также ряд других результатов натолкнули некоторых исследователей на мысль о том, что важную роль в механизме формирования поверхности КС играет пластическая деформация $[1,3,5,9,11]$. Было сделано предположение о том, что в локальном объеме перед устьем трещины водород приводит не к охрупчиванию, а, фактически, к пластификации материала, например, за счет снижения барьера Пайерлса и уменьшения эффективного радиуса упругого взаимодействия дислокаций [12]. Подразумевается, что в результате этого ВАР происходит по видоизмененному механизму вязкого разрушения, т. е. путем образования и слияния пор, которые, однако, имеют нанометровые размеры $[1,5,11]$. В качестве подтверждения данной теории, в частности, приводятся изображения, полученные при помощи атомносиловой микроскопии, демонстрирующие наноямки на поверхности фасеток КС [11].

Другое распространенное мнение заключается в том, что поверхность КС является результатом хрупкого разрушения, которое осуществляется по механизму ИС, в то время как следы вязкого разрушения в изломе - лишь следствие сопутствующей пластической деформации $[2,6]$. Предполагается, что водород может провоцировать скол за счет снижения силы сцепления атомов в кристаллической решетке [2] или за счет блокирования эмиссии дислокаций из устья трещины [6]. Одним из доводов в пользу данной теории является тот факт, что в монокристаллах сплава $\mathrm{Fe}-3 \% \mathrm{Si}$ поверхность КC имеет кристаллографическую ориентацию $\{001\}[1,2,6]$, т. е. совпадающую с плоскостями скола в альфа-железе. Однако, например, в чистом железе и низкоуглеродистой стали фасетки КС чаще имеют ориентацию $\{011\}$ и $\{112\}[3,4,13]$. Более того, в работах $[9,14]$ показано, что зачастую трещины КС, вообще, могут сильно отклоняться от конкретных кристаллографических плоскостей. При помощи конфокальной лазерной сканирующей микроскопии (3D) в нашей предыдущей работе [7] было показано, что фасетки КС в наводороженной низкоуглеродистой стали марки S235JR имеют в два раза меньший средний угол разориентировки, но в три раза большую кривизну, по сравнению с фасетками ИС в той же стали, испытанной в жидком азоте. Для того чтобы исключить возможное влияние разной степени пластической деформации, которая была выше у образца, испытанного на воздухе после наводороживания, чем у образца без водорода, испытанного в жидком азоте, в последующей работе [8] был получен излом той же стали, который одновременно содержал фасетки обоих типов. При этом, было установлено наличие четкой границы между фасетками ИС и КС а также качественные отличия между их топографией. Тем не менее, из-за недостаточного количества фасеток КС в изломе предварительно наводороженного образца, проведение статистически достоверного анализа количественных характеристик фасеток было невозможным. Для того чтобы восполнить данный пробел, в настоящем исследовании было использовано in-situ наводороживание в процессе растяжения надрезанного образца до заданной длины трещины, которое, в сочетании с последующим доломом образца в жидком азоте, позволило получить излом, содержащий достаточное количество фасеток ИС и КС. При этом целью данной работы было статистически 
достоверно установить количественные характеристики поверхностей разрушения низкотемпературного истинного скола и, водородо-ассистированного квазискола в низкоуглеродистой стали.

\section{2. Материал и методика исследования}

В качестве модельного материала исследования использовали промышленную низкоуглеродистую сталь марки S235JR (близкий отечественный аналог сталь 15), которая применялась в наших предыдущих работах [7-9] (хим. состав представлен в Табл. 1). Из горячекатаного листа указанной стали вдоль направления прокатки при помощи электроискрового станка были вырезаны прямоугольные образцы размером $60 \times 11 \times 2.5$ мм с центральным симметричным сквозным надрезом, ориентированным перпендикулярно оси растяжения образца (см. врезку на Рис. 1а). Образцы подвергали шлифовке при помощи наждачной бумаги до номера 2500 , затем отжигали в вакууме при $950^{\circ} \mathrm{C}$ в течение 1 часа, после чего проводили полировку при помощи суспензий с частицами 3 и 1 мкм и травление в растворе $0.5 \% \mathrm{HNO}_{3}$ в ультразвуковой ванне.

Перед установкой образца в захваты разрывной машины на нем закрепляли электролитическую ячейку для наводороживания с платиновым анодом в виде проволочной спирали. Непосредственно перед началом растяжения ячейку заполняли электролитом состава $5 \% \mathrm{H}_{2} \mathrm{SO}_{4}+1.5$ г/л тиомочевины. При этом с электролитом контактировала только центральная часть образца площадью $5.4 \mathrm{~cm}^{2}$. Электролиз с плотностью тока $5 \mathrm{~mA} / \mathrm{cm}^{2}$ начинали одновременно с испытанием на растяжение, которое проводили при скорости движения траверсы разрывной машины 0.1 мм/мин (диаграмма растяжения указана стрелкой (1) на Рис. 1a). Растяжение и электролитическое наводороживание прекращали в тот момент, когда трещина, распространявшаяся из надреза, достигала длины равной 1.2 мм, т.е., примерно, одной трети сечения образца. Внешний вид боковой поверхности образца с такой трещиной представлен Рис. 1b. Характерная точка на диаграмме растяжения, соответствующая такой длине трещины и отмеченная стрелкой (2) на Рис. 1a, была установлена экспериментально, путем нескольких предварительных испытаний. После остановки испытания образец разгружали (стрелка (3) на Рис. 1а) и повторно испытывали до разрушения в жидком азоте при скорости движения траверсы 1 мм/мин (стрелка (4) на Рис. 1a).

Для исследования излома и боковой поверхности образца использовали сканирующий электронный микроскоп (СЭМ) JEOL JCM-6000 и КЛСМ Lext OLS4000 (Olympus). При помощи КЛСМ были получены топографические карты нескольких участков излома в области, как ИС, так и КС. Площадь каждого участка составляла $256 \times 256$ мкм. С использованием полученных таким образом 2D изображений и массивов высот, а также программного обеспечения, разработанного в НИТУ «МИСиС», были рассчитаны углы разориентировки и кривизна более 500 фасеток каждого типа. Принцип работы данного ПО заключается в следующем: 1) на $2 \mathrm{D}$ изображении, полученном с помощью КЛСM, оператор вручную выделяет границы всех фасеток; 2) затем для каждой выделенной фасетки ПО находит соответствующий фрагмент массива высот, производит егоаппроксимациюплоскостьюинаходиткоэффициенты уравнения этой плоскости; 3) с использованием полученных данных вычисляются углы между смежными фасетками - углы разориентировки $\alpha$, а также дисперсия

Табл. 1. Химический состав стали S235JR.

Table 1. Chemical composition of the steel S235JR.

\begin{tabular}{|c|c|c|c|c|c|c|c|c|c|c|}
\hline Элемент / Element & $\mathrm{C}$ & $\mathrm{Cu}$ & $\mathrm{Si}$ & $\mathrm{Mn}$ & $\mathrm{P}$ & $\mathrm{S}$ & $\mathrm{Cr}$ & $\mathrm{Ni}$ & $\mathrm{Al}$ & $\mathrm{Fe}$ \\
\hline Bec.\% / Wt.\% & 0.129 & 0.067 & 0.02 & 0.42 & 0.019 & 0.015 & 0.05 & 0.007 & 0.028 & Ocнова / Balance \\
\hline
\end{tabular}

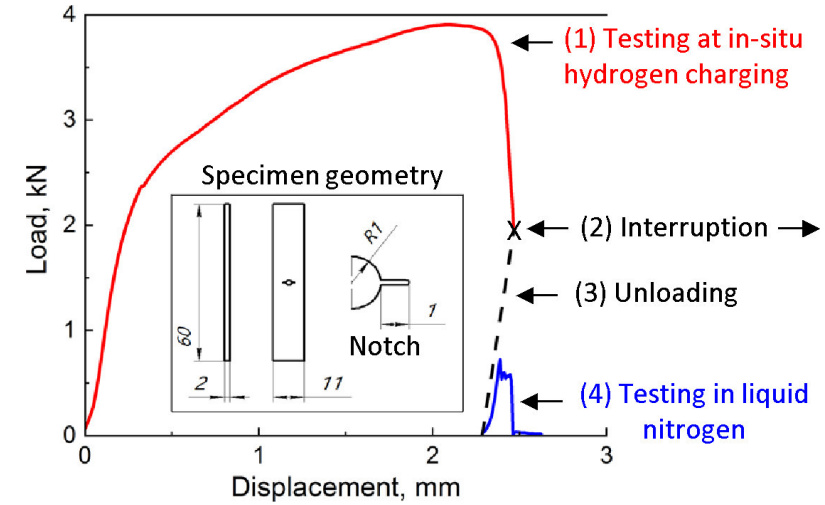

a

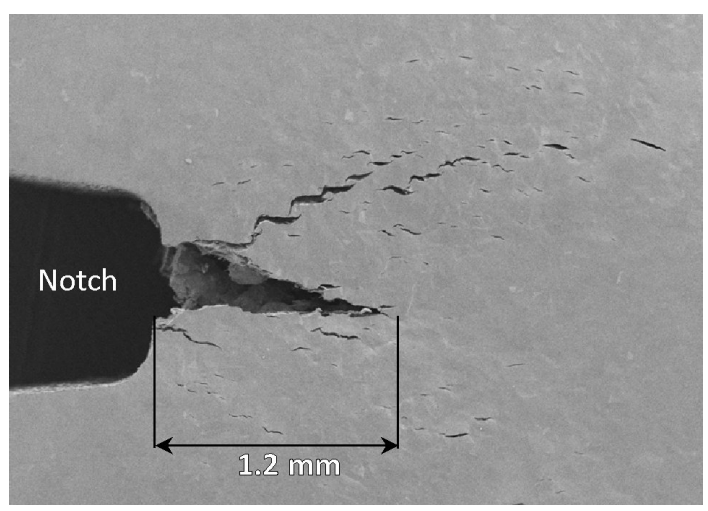

$\mathrm{b}$

Puc. 1. (Color online) Иллюстрация методики эксперимента, включающая: геометрию применявшихся образцов (а, врезка), диаграммы нагружения (а) и, полученный при помощи СЭМ, снимок боковой поверхности образца после остановки испытания на растяжение при in-situ наводороживании в точке, отмеченной стрелкой (2) на диаграмме нагружения (b).

Fig. 1. (Color online) Illustration of the experimental procedure, showing the geometry of the specimens used (a, inset), the loading diagram (a) and the SEM image of the specimen side surface after interruption of the tensile test with in-situ hydrogen charging at the point indicated by the arrow (2) on the tensile diagram (b). 
аппроксимации фасетки плоскостью $D$, рассчитанная как среднеквадратичное отклонение рельефа поверхности фасетки от плоскости. Величину $D$ считали пропорциональной кривизне фасетки. Более подробно детали данной методики описаны в наших предыдущих работах $[7,15]$.

\section{3. Результаты и обсуждение}

Общий вид излома, полученного в результате испытания, представлен на Рис. 2. На изображении четко различимы два участка поверхности разрушения, очевидно, имеющие принципиально разную морфологию. Первый участок, начинающийся от надреза, имеет длину около 1.2 мм, которая совпадает с длиной трещины на боковой поверхности образца, после его испытания при in-situ наводороживании, но до испытания в жидком азоте (Рис. 1b). Следовательно, данный участок был образован в результате ВАР, которое происходило в процессе растяжения образца при in-situ наводороживании. В работе [9] было показано, что in-situ наводороживание позволяет полностью подавить обычное вязкое разрушение. В полном соответствии с этими результатами, первый участок излома представлен исключительно фасетками КС с характерной морфологией, показанной на Рис. 3 с, без признаков классического вязкого ямочного рельефа. На расстоянии 1.2 мм от надреза, наблюдается четкая граница между КС и ИС (Рис. 2 и $3 \mathrm{a}, \mathrm{d})$. При этом, какой-либо переходной зоны между двумя участками излома не наблюдается (Рис. 2 -4). При анализе поверхности разрушения образца в масштабе всего излома, можно заметить, что относительно плоский рельеф поверхности разрушения на участке КС резко приобретает существенно более шероховатый и угловатый характер при переходе через границу с участком ИС (см. профиль на Рис. 2). В то же время, если поверхность разрушения рассматривается в масштабе нескольких фасеток, то видно, что фасетки КС имеют искривленный шероховатый профиль, тогда как фасетки ИС гораздо более плоские, хотя и ориентированы друг к другу под большими углами (Рис. 4). Кроме того, в сравнении с фасетками КС (Рис. $3 \mathrm{a}, \mathrm{c}, \mathrm{d})$, фасетки ИС имеют более правильное строение с типичным речным узором, а также - более четкие границы (Рис. 3 a, b, d).

Количественный фрактографический анализ показал, что средний угол разориентировки а у фасеток КС примерно в 1.5 раза ниже, а кривизна $D$ в 3 раза выше, чем у фасеток ИС (Табл. 2). Стоит отметить, что в предыдущей работе [7], в которой определение количественных характеристик фасеток каждого типа производилось на разных образцах, значения а для фасеток ИС и КС отличались в 2 раза. При этом величина $а$ для фасеток КС в [7] была немного ниже, а для фасеток ИС, наоборот немного выше, чем в настоящей работе. Кривизна фасеток в предыдущей работе также как и в настоящем исследовании отличалась в три раза, но абсолютные значения $D$ для обоих типов фасеток были немного ниже. Вероятно, данные изменения могут быть связаны с влиянием пластической деформации и полей напряжения от надреза. В процессе испытания при in-situ наводороживании, еще до образования магистральной трещины, впереди надреза образуется пластическая зона. Предположительно, остаточная деформация в данной зоне может быть выше, чем остаточная деформация образца, который был испытан в жидком азоте в работе [7]. Согласно [10] пластическая деформация может приводить к уменьшению углов разориентировки фасеток ИС, а также к увеличению их кривизны. В то же время в работе [9] было показано,

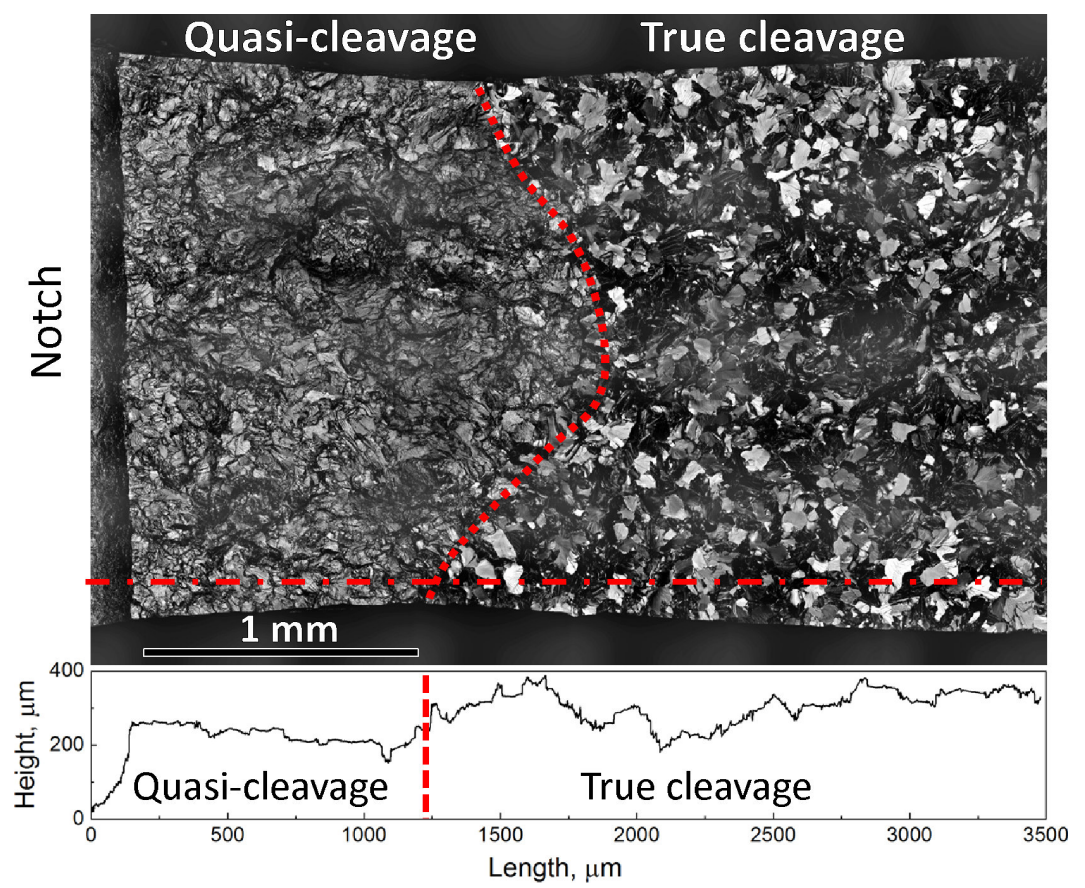

Pис. 2. (Color online) Общий вид излома и профиль его поверхности вдоль горизонтальной штрихпунктирной линии. КЛСМ. Fig. 2. (Color online) A view of the whole fracture surface and its profile along the horizontal dot-and-dash line: CLSM analysis. 

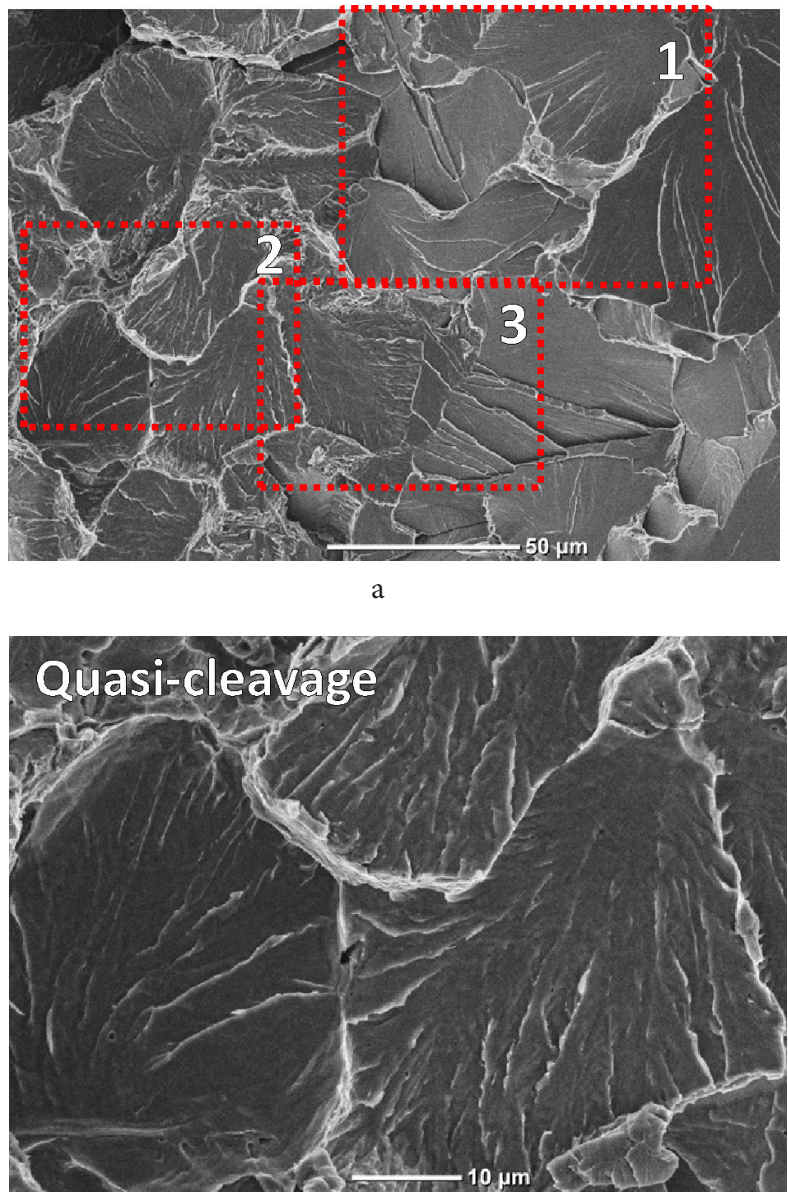

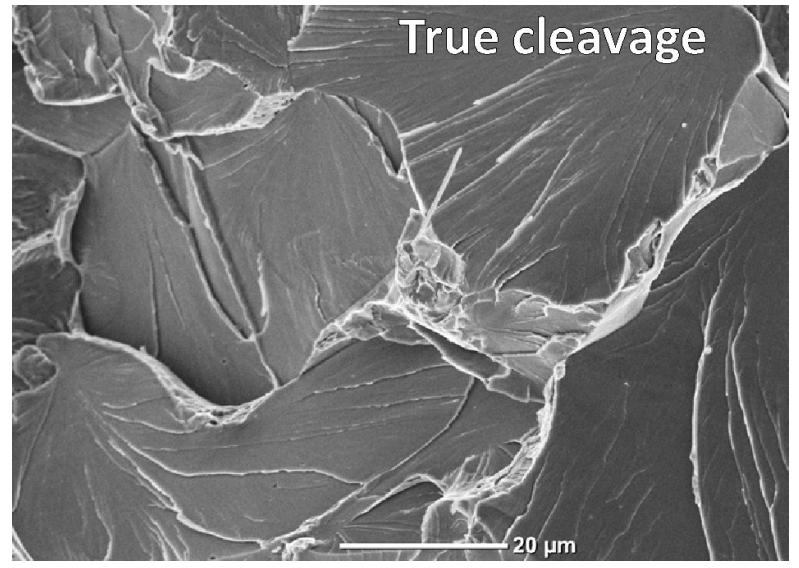

$\mathrm{b}$

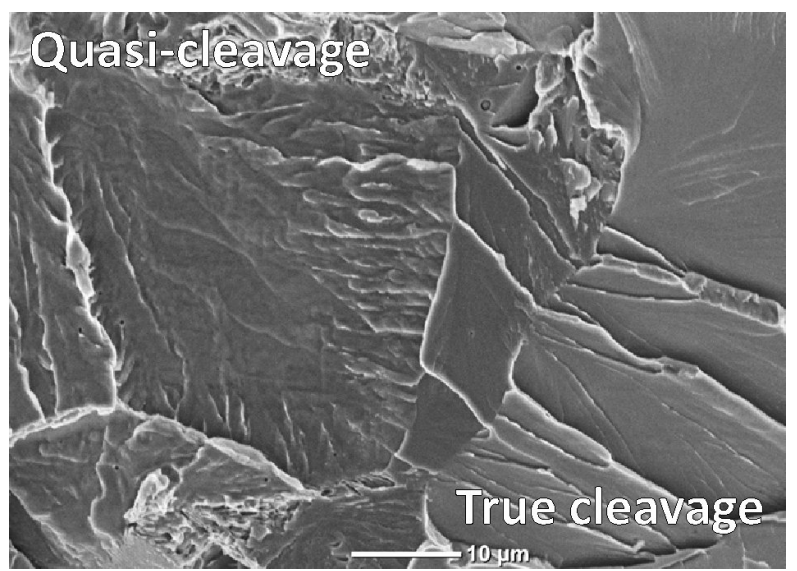

d

Pис. 3. Фрактографические снимки в области перехода от участка квазискола к истинному сколу: общий вид переходной зоны (а), соответственно области 1, 2 и 3 на Рис. 3 а при большем увеличении (b), (c) и (d). СЭМ.

Fig. 3. SEM fractographic images in the transition region between quasi-cleavage and true cleavage morphologies: general view of the transition region (a), regions marked by 1, 2 and 3 in Fig. 3 a, respectively, imaged at higher magnification (b), (c) and (d).

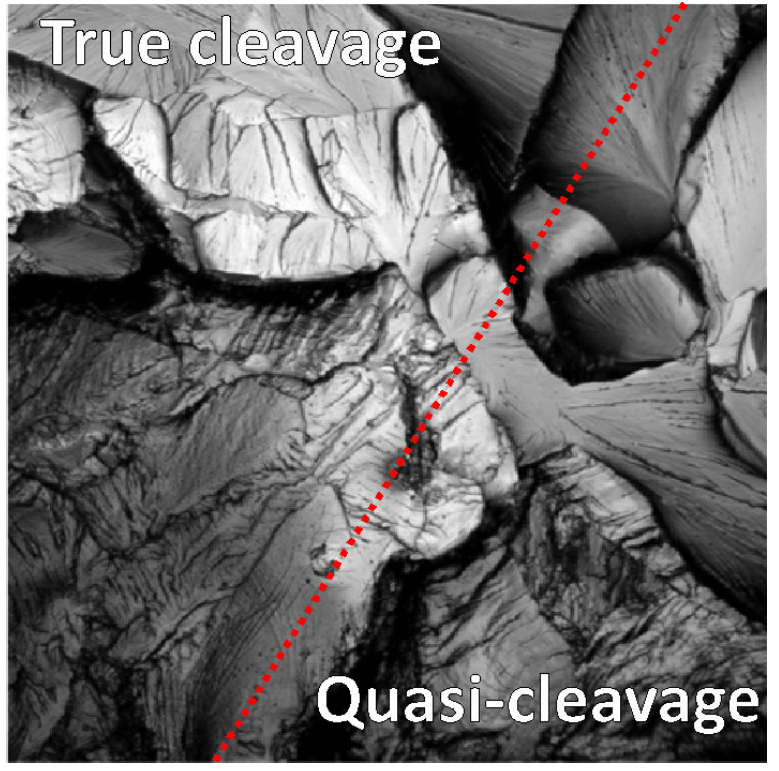

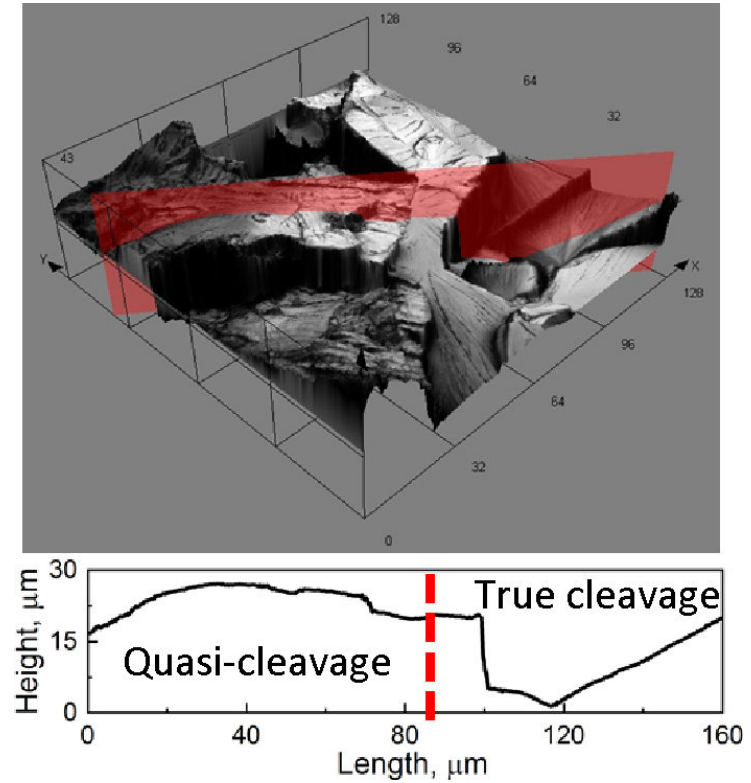

$b$

Pис. 4. (Color online) Поверхность разрушения и ее профиль в области перехода от квазискола к истинному сколу. КЛСМ. Вид сверху (a), изометрия и профиль поверхности вдоль красной секущей плоскости (b).

Fig. 4. (Color online) The fracture surface and its profile in the transition region between quasi-cleavage and true cleavage. CLSM. The top view (a), the isometric view with surface profile along the red cross-section plane (b). 
Табл. 2. Количественные характеристики фасеток.

Table 2. Quantitative characteristics of facets.

\begin{tabular}{|c|c|c|c|c|}
\hline & \multicolumn{2}{|c|}{$\begin{array}{c}\text { Средний угол разориентировки фасеток } \alpha, \text { град. } \\
\text { Average misorientation angle of facets } \alpha \text {, deg. }\end{array}$} & $\begin{array}{c}\text { Кривизна (дисперсия аппроксимации) фасеток } D, \text { мкм } \\
\text { Curviness (dispersion of approximation) of facets } D, \mu \mathrm{m}^{2}\end{array}$ \\
\hline & $\begin{array}{c}\text { Квазискол } \\
\text { Quasi-cleavage }\end{array}$ & $\begin{array}{c}\text { Скол } \\
\text { Cleavage }\end{array}$ & $\begin{array}{c}\text { Квазискол } \\
\text { Quasi-cleavage }\end{array}$ & $\begin{array}{c}\text { Скол } \\
\text { Cleavage }\end{array}$ \\
\hline $\begin{array}{c}\text { Настоящая работа } \\
\text { Рresent study }\end{array}$ & 23.2 & 35.7 & 1.27 & 0.46 \\
\hline$[7]$ & 19.1 & 39.5 & 1.01 & 0.28 \\
\hline
\end{tabular}

что поля деформации и напряжений от надреза при in-situ наводороживании могут оказывать существенное влияние на путь трещин КС, приводя к его искривлению, что, по-видимому, должно вызвать, как увеличение кривизны самих фасеток КС, так и рост среднего угла их разориентировки. Поскольку в предыдущей работе использовались гладкие образцы без надреза, то наличие надреза у образцов в настоящей работе может объяснить увеличение значений а и $D$ у фасеток КС. Несмотря на вышесказанное, отличия между количественными характеристиками фасеток ИС и КС значительны, и не могли быть вызваны только лишь влиянием пластической деформации. В противном случае, в изломе наблюдался бы плавный переход от одного типа поверхности разрушения к другому, а не резкая граница, которая обнаружена в настоящей работе, а также в работе [8]. Следовательно, существенно более низкий угол разориентировки, а также более высокая кривизна фасеток КС по сравнению с фасетками ИС, должны быть обусловлены принципиальными отличиями в механизме образования данных фасеток. Таким образом, водородоассистированное растрескивание низкоуглеродистой стали, сопровождающееся формированием поверхности разрушения с морфологией КС, вряд ли, может быть объяснено с позиции теорий, подразумевающих, что водород тем или иным образом провоцирует рост трещин по механизму ИС.

\section{4. Выводы}

1. Образец отожженной низкоуглеродистой стали, в котором была выращена трещина в процессе его растяжения при электролитическом in-situ наводороживании, и затем доломанный в жидком азоте, имеет излом, содержащий участки квазискола и истинного скола с четкой границей между ними без переходной зоны.

2. Средний угол разориентировки фасеток квазискола, образованных в результате водородо-ассистированного растрескивания, составляет $23.2^{\circ}$, что в 1.5 раза ниже, чем у фасеток истинного скола, образованных в том же образце в результате хладноломкости, средний угол разориентировки которых составляет $35.7^{\circ}$. При этом кривизна фасеток квазискола в 3 раза выше, чем у фасеток скола.

3. Более низкий угол разориентировки и более высокая кривизна фасеток квазискола по сравнению с фасетками истинного скола обусловлены принципиаль- ными отличиями механизмов образования данных типов фасеток.

4. Механизм истинного скола не играет ключевой роли в механизме образования фасеток квазискола в охрупченной водородом низкоуглеродистой стали.

Благодарности /Acknowledgements. Работа выполнена при финансовой поддержке Российского научного фонда (проекm 19-79-00188). / Financial support from the Russian Science Foundation through the grant-in-aid No. 19-79-00188 is gratefully appreciated.

\section{Литература/References}

1. S. P. Lynch. Corros. Rev. 30, 63 (2012). Crossref

2. X. Chen, W. W. Gerberich. Metall. Trans. A. 22, 59 (1991). Crossref

3. F. Nakasato, I. Bernstein. Metall. Mater. Trans. A. 9, 1317 (1978). Crossref

4. K. Okada, A. Shibata, Y. Takeda, N. Tsuji. Int. J. Hydrogen Energy. 43, 11298 (2018). Crossref

5. M.L. Martin, J.A. Fenske, G.S. Liu, P. Sofronis, I. M. Robertson. Acta Mater. 59, 1601 (2011). Crossref

6. D. Birenis, Y. Ogawa, H. Matsunaga, O. Takakuwa, J. Yamabe, Ø. Prytz, A. Thøgersen. Mater. Sci. Eng. A. 756, 396 (2019). Crossref

7. E. Merson, A.V. Kudrya, V.A. Trachenko, D. Merson, V. Danilov, A. Vinogradov. Mater. Sci.Eng. A. 665, 35 (2016). $\underline{\text { Crossref }}$

8. E.D. Merson, V.A. Poluyanov, D.L. Merson, A. Y. Vinogradov. Met. Sci. Heat Treat. 61, 191 (2019). Crossref

9. E.D. Merson, P.N. Myagkikh, V.A. Poluyanov, D. L. Merson, A. Vinogradov. Eng. Fract. Mech. 214, 177 (2019). Crossref

10. M. A. Shtremel. Fracture. Book 2. Moscow, MISIS (2015) 975 p. (in Russian) [M.А. Штремель. Разрушение. Книга 2. Москва, МИСИС (2015) 975 с.]

11. T. Neeraj, R. Srinivasan, J. Li. Acta Mater. 60, 5160 (2012). Crossref

12. M.L. Martin, M. Dadfarnia, A. Nagao, S. Wang, P. Sofronis. Acta Mater. 165, 734 (2019). Crossref

13. N. Takano, K. Kidani, Y. Hattori, F. Terasaki. Scr. Metall. Mater. 29, 75 (1993). Crossref

14. M.L. Martin, I. M. Robertson, P. Sofronis. Scr. Mater. 59, 3680 (2011). Crossref

15. E. Merson, V. Danilov, D. Merson, A. Vinogradov. Eng. Fract. Mech. 183, 147 (2017). $\underline{\text { Crossref }}$ 